\title{
A method to assess the failure probabilities of river levees based on Elicitation, Calibration, Aggregation, and Debiasing of expert judgement (leCAD)
}

\author{
Laurent Peyras ${ }^{2}$, Claudio Carvajal ${ }^{2}$, Youssef Diab ${ }^{1,3}$, Michel Hathout ${ }^{1,2}$, Marc Vuillet ${ }^{1}$ \\ ${ }^{1}$ Lab’Urba, Ecole des Ingénieurs de la Ville de Paris, Université Paris-Est, 80 rue Rébeval, 75019 Paris - France \\ ${ }^{2}$ INRAE, Aix-Marseille Univ, RECOVER, 3275 route Cézanne - CS 40061 - 13182 Aix en Provence - France \\ ${ }^{3}$ Lab'Urba, Université Paris-Est (Marne-la-Vallée), bureau J29, Bât Lavoisier, 5 Bvd Descartes 77420 Champs-sur-Marne - France
}

\begin{abstract}
Studies carried out to analyse the risks of levees must include an evaluation of the probabilities of occurrence of different failure mechanisms (overflow, internal erosion, sliding and scouring). The probabilistic quantitative evaluation of these mechanisms remains difficult due to often insufficient input data, the natural variability of the materials, structures of very long length, the availability of mechanical models for certain failure mechanisms, and the random nature of the stresses involved. This makes it necessary to call for expert judgement to evaluate the probabilities of failure. However, expert judgement is generally associated with a qualitative and subjective dimension, and it comprises biases liable to impair the capacities of an expert to elicit their evaluations. This article proposes an approach to processing expert judgement that includes the modalities of Individual expert Elicitation, Calibration, Aggregation, and Debiasing of expert judgement (IeCAD). This IeCAD approach has been developed for river levees in view to correcting biased expert evaluations in the case of evaluating the failure probability of structures.
\end{abstract}

\section{Introduction}

River levees are structures that are raised above the natural level of the land in view to protecting naturally floodable areas (Mériaux et al., 2007). Evaluating the safety of levees is a major challenge for the managers of these structures in order to predict a risk of failure (Kolen et al., 2013). Moreover, many regulations require the evaluation of the reliability of structures in a probabilistic framework, in order to demonstrate levee failure risks (Ciria et al., 2013 ; Peyras et al., 2015).

Evaluating the safety of river levees in a probabilistic framework is difficult due to the scarcity of available data. Due to complex levee failure modes (Simm et al., 2012), the different sources of data for levee assessments (Van der Meij et al., 2012), and the uncertainty on the data available (Vuillet et al., 2013), a high level of expertise is required to determine levee reliability for the failure modes (Mériaux and Royet, 2007). Most of the failure mechanisms - internal erosion, scouring - cannot be evaluated in a probabilistic framework without calling on expert judgement. This leads to privileging expert judgement when evaluating a failure probability.

The objective of this study is to propose a probabilistic approach to evaluate the safety of levees based on expert judgement. The study proposes specific elicitation, calibration and debiasing methods to process expert evaluations and thus reduce the biases intrinsic to expert judgement.

The methods of treating expert judgement are mainly dealt with in the statistical literature, with applications in economics (Lichtendahl et al., 2013), and industry (Cooke, 1991; Clemen and Lichtendahl, 2002). They have been developed to reduce biases when employing expert judgement and consist of three main modalities for dealing with bias: i) elicitation of expert opinion, by focusing on developing the best modalities for collecting opinions in view to eliciting the most precise expert judgement possible (Vick, 2002); ii) calibration of expert judgements (Cooke, 1991), including the weighting of opinions as a function of their pertinence, and their aggregation (Lichtendahl et al., 2013); iii) debiasing expert judgement, aimed at applying mathematical corrections to expert evaluations (Clemen et al., 2002).

Regarding civil engineering, there are few works that focus on treating expert judgement and reducing bias. Peyras et al. (2006) suggested employing expert judgement to measure the occurrence of function loss. Nonetheless, the subjective probabilities provided by the experts were not subjected to any specific protocol relating to their collection or treatment. Vuillet et al. (2013) proposed modalities for eliciting expert judgement aimed at reducing biases.

\footnotetext{
${ }^{a}$ Corresponding author: laurent.peyras@inrae.fr
}

DOI 10.3311/FLOODRisk2020.24.8 
The paper presents the results of our study relating to the calibration and aggregation procedures proposed for river levees. However the theoretical developments are not developed in this paper. The complete method, their methodological basis and the complete results are given in (Hathout et al, 2020).

The paper first presents the development of a protocol for eliciting expert opinions in the form of failure probabilities relating to failure mechanisms. Then, a calibration and aggregation model is proposed for the opinions expressed by a panel of experts. Lastly, the article presents the development of an approach used to debias the opinion of the expert panel. The approach developed is applied to the case of an existing levee.

\section{Approach to evaluating failure probabilities by expert judgement}

\subsection{Principles}

The principle of the approach is based on the comparison between the calibration variables whose true values are known through numerical calculation and the variables of interest whose real values are unknown and determined by expert judgement. The proposed IeCAD (Individual elicitation, Calibration, Aggregation, Debiasing) approach comprises three steps of treating expert judgement (Figure 1):

- The elicitation by expert judgement of the failure probabilities of levee failure mechanisms,

- The calibration and aggregation of the opinions of different experts in view to aggregating them,

- The debiaising of expert opinions in order to process the biases of over- and under-confidence liable to impair the calibrated and aggregated expert evaluations:

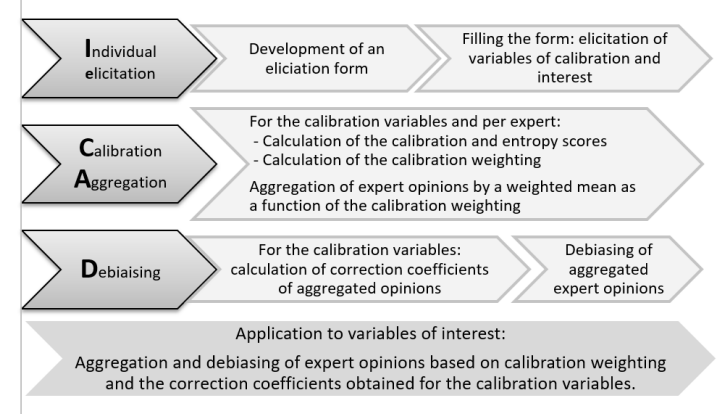

Figure 1. Explanatory chart of the IeCAD approach developed.

\subsection{Identification of the calibration variables and the variables of interest}

We adopted as variable of interest the failure probability of levee cross-sections with respect to sliding, internal erosion and scouring failure mechanisms.

The calibration variables must be of the same nature as the variables of interest to permit calibration and debiasing (Cooke, 1991). We adopted the failure probability of the cross-sections of levees with respect to the sliding failure mechanism. The probabilistic calibration variables are determined by expert judgment on the one hand, and by numerical calculation on the other. The comparison between the expert and numerical evaluation allows determining the calibration weighting given to the expert opinions of each expert of the panel and the correction coefficients given to the expert biases.

To determine the calibration variables by numerical calculation, we search the failure probability of the sliding failure mechanism using a mechanical-probabilistic model based on the limit equilibrium and in which the resistive properties of the materials are modelled by probabilistic laws (Mouyeaux et al., 2018). It is then possible to use Monte Carlo simulations to obtain a probabilistic distribution of the safety factor and calculate the failure probability of the cross-section studied.

\subsection{Elicit expert opinions for river levees}

Implementing the approach to eliciting expert opinions starts with identifying a panel with several experts whose activities are directly linked to river levees (Koehler and Harvey, 2004).

Each of the experts of the panel is questioned individually on the failure probabilities of several levee cross-sections to be evaluated with respect to different failure mechanisms. A questionnaire form used to collect the expert judgement was developed to this end. For each calibration variable and variable of interest to be elicited, the form contains three main items (Figure 2): the question, the information and the response:

- The question asked to the experts concerns the sliding failure mechanism: "Given all the information available, what is the probability $P f c$ that the levee will fail due to sliding of the downstream slope if a flood that reaches the crest of the levee occurs?"

- The information are the geometric, hydraulic and geotechnical data, the standard cross-section to be evaluated, the probability laws of the geotechnical characteristics of the cross-section, the data from the deterministic analysis represented by the safety factor SF related to the sliding failure mechanism.

- The response of the expert elicitations in the form of an uncertainty interval and a most likely value:

- an uncertainty interval [quantile 5\%, quantile $95 \%$,

- $\quad$ the most likely value contained in the uncertainty interval elicited. 


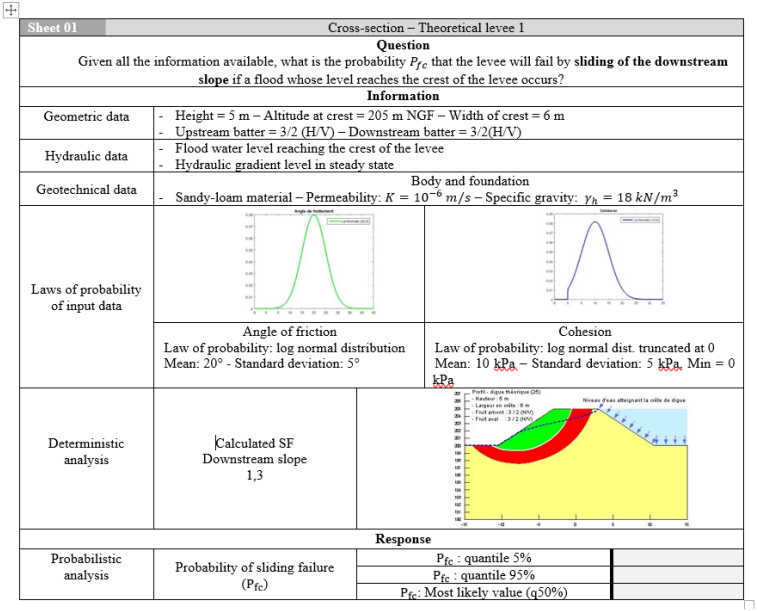

Figure 2. Example of a levee cross-section sheet taken from the questionnaire form.

\subsection{Expert opinion calibration, aggregation and debiasing approach}

The approach to calibrating the panel experts' opinions is based on Cooke's (1991) model. It consists in evaluating and weighting the panel experts' opinions in relation to calibration variables whose real values are known. Cooke's (1991) model allows calculating an individual calibration weight $w_{e}$ for each expert and the relative calibration weight $w_{e}^{\prime}$ in relation to all the experts of the panel, since the closer this expert's elicitations are to reality, the higher the calibration weight assigned to them is. We determine the calibration weight on the basis of the calibration score $C_{\mathrm{e}}$ and the entropy score $K_{\mathrm{e}}$ according to the formulas proposed by (Cooke, 1991).

The debiasing approach aims to apply mathematical corrections to the panel's expert evaluations, in order to get as close as possible to the calibration variables. The objective is to quantitatively identify the trend of the expert panel's opinions to under- or over- confidence liable to impair the estimation of the most likely value and the uncertainty interval, and then to apply a mathematical correction. In this purpose, we implement an expert opinion debiasing approach based on the model of Clemen and Lichtendahl (2002), consisting in determining three correction coefficients by iterative calculation.

\section{Application to a River levees}

The levee studied is an earth-fill levee 5500 meters long raised to protect a town in France. The height of the levee varies from 1 to $6 \mathrm{~m}$ (Figure 3).
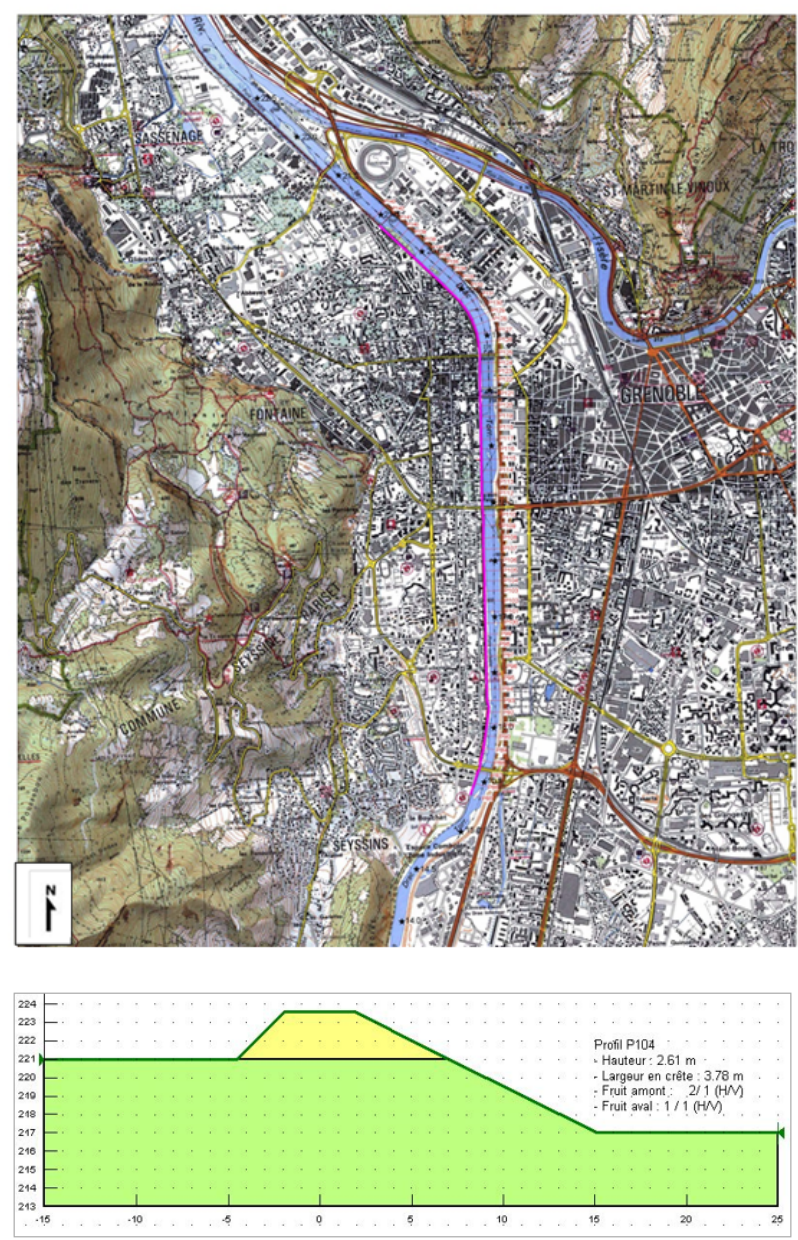

Figure 3. Aerial view and standard cross-section of the levee studied.

The approach developed was implemented by a panel of 6 engineers having different professional backgrounds. The calibration variables (variables no.1 to 30) correspond to failure probabilities associated with the sliding mechanism for 30 cross-sections, permitting a robust statistical analysis.

The study was applied to 30 variables of interest (variables no.31 to 60 - Table 1). Variables of interest no.31 to 40 correspond to failure probabilities of the sliding mechanism for which real values are obtained from a numerical mechanical-probabilistic calculation. Variables of interest no.41 to 60 correspond to failure probabilities with respect to internal erosion and scouring mechanisms.

\begin{tabular}{|c|c|c|c|c|}
\hline \multirow{2}{*}{$\begin{array}{c}\text { Failure } \\
\text { mechanism }\end{array}$} & \multicolumn{2}{|c|}{ Calibration variables } & \multicolumn{2}{|c|}{ Variables of interest } \\
\hline & $\begin{array}{l}\text { Number of } \\
\text { variables }\end{array}$ & $\begin{array}{c}\text { no. of } \\
\text { variable }\end{array}$ & $\begin{array}{l}\text { Number of } \\
\text { variables }\end{array}$ & $\begin{array}{c}\text { no. of } \\
\text { variable }\end{array}$ \\
\hline Sliding & 30 & no. $1-30$ & 10 & no. $31-40$ \\
\hline Internal erosion & - & - & 10 & no. $41-50$ \\
\hline Scouring & - & - & 10 & no. $51-60$ \\
\hline
\end{tabular}

Table 1. Table summarising the calibration variables and the variables of interest used in the approach. 


\subsection{Application and results of the expert opinion elicitation phase}

Figure 4 presents the elicitations of expert no.1 relating to failure probabilities $\left(\mathrm{P}_{\mathrm{fc}}\right)$ regarding 3 failure mechanisms, and Table 2 shows the distribution the true known values of the calibration variables in the interquantile intervals elicited by the 6 experts for the cross-sections no. 1 to 30 .

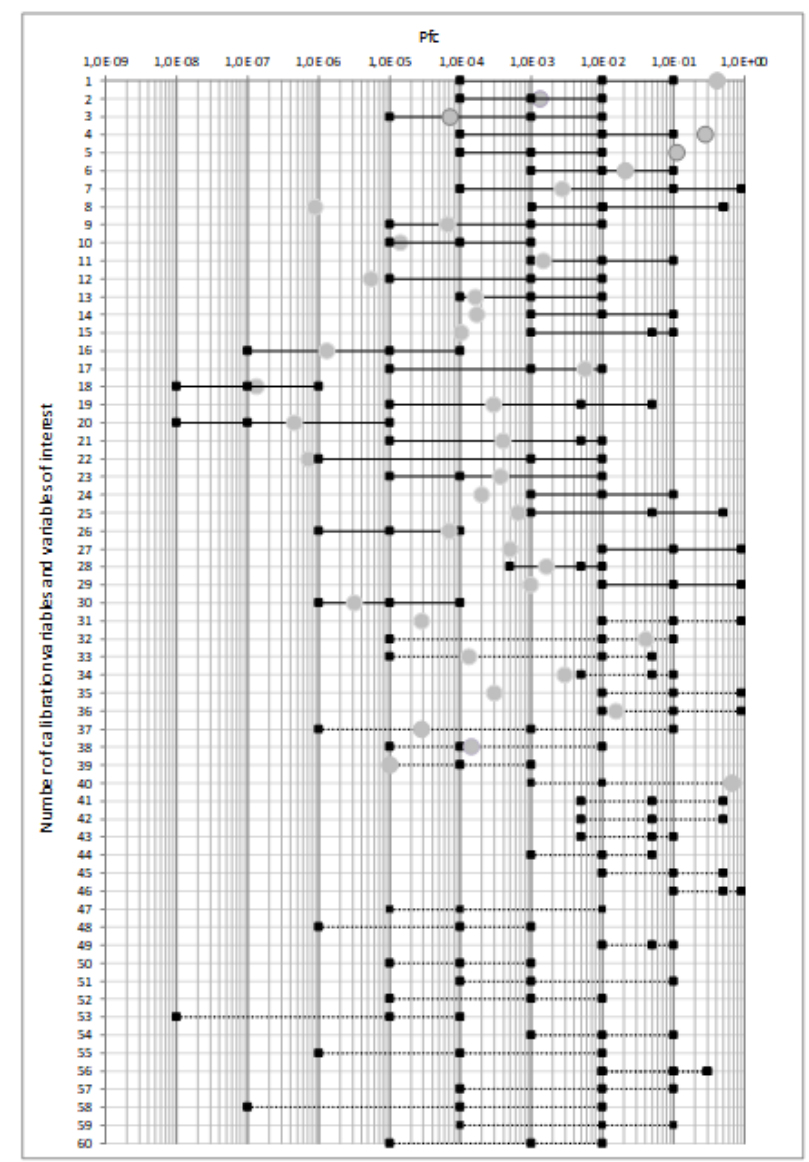

Figure 4. Elicitations of expert no. 1 relating to calibration variables.

\begin{tabular}{|c|c|c|c|c|c|}
\hline \multicolumn{6}{|c|}{ Calibration variable (no.1 to 30 ) } \\
\hline \multicolumn{2}{|c|}{$\begin{array}{c}\text { Interquantile } \\
\text { interval }\end{array}$} & {$[0 \%, 5 \%]$} & {$[\mathbf{5} \%, \mathbf{5 0} \%]$} & {$[\mathbf{5 0} \%, \mathbf{9 5} \%]$} & {$[95 \%, 100 \%]$} \\
\hline \multicolumn{6}{|c|}{ Expert no.1 } \\
\hline \multirow{2}{*}{$\begin{array}{l}\left(\mathbf{P}_{\mathrm{fc}}\right) \\
\text { (no.1 to } \\
\text { no. } 30 \text { ) }\end{array}$} & $\mathrm{n}$ & 9 & 11 & 7 & 3 \\
\hline & $\%$ & $30 \%$ & $37 \%$ & $23 \%$ & $10 \%$ \\
\hline \multicolumn{6}{|c|}{ Expert no.2 } \\
\hline \multirow{2}{*}{$\begin{array}{l}\left(\mathbf{P}_{\mathrm{fc}}\right) \\
\text { (no. 1 to } \\
\text { no. } 30 \text { ) }\end{array}$} & $\mathrm{n}$ & 9 & 10 & 10 & 1 \\
\hline & $\%$ & $30 \%$ & $33 \%$ & $33 \%$ & $3 \%$ \\
\hline \multicolumn{6}{|c|}{ Expert no.3 } \\
\hline \multirow{2}{*}{$\begin{array}{l}\left(\mathbf{P}_{\mathrm{f}_{\mathrm{f}}}\right) \\
\text { (no. } 1 \text { to } \\
\text { no. } 30)\end{array}$} & $\mathrm{n}$ & 10 & 5 & 7 & 8 \\
\hline & $\%$ & $33 \%$ & $17 \%$ & $23 \%$ & $27 \%$ \\
\hline \multicolumn{6}{|c|}{ Expert no.4 } \\
\hline \multirow{2}{*}{$\begin{array}{l}\left(\mathbf{P}_{\mathrm{fc}}\right) \\
\text { (no.1 to } \\
\text { no.30) }\end{array}$} & $\mathrm{n}$ & 10 & 8 & 8 & 4 \\
\hline & $\%$ & $33 \%$ & $27 \%$ & $27 \%$ & $13 \%$ \\
\hline \multicolumn{6}{|c|}{ Expert no.5 } \\
\hline \multirow{2}{*}{$\begin{array}{l}\left(\mathbf{P}_{\mathrm{fc}}\right) \\
\text { (no. } 1 \text { to } \\
\text { no. } 30 \text { ) }\end{array}$} & $\mathrm{n}$ & 0 & 4 & 15 & 11 \\
\hline & $\%$ & $0 \%$ & $13 \%$ & $50 \%$ & $37 \%$ \\
\hline \multicolumn{6}{|c|}{ Expert no.6 } \\
\hline \multirow{2}{*}{$\begin{array}{l}\left(\mathbf{P}_{\mathrm{fc}}\right) \\
\text { (no. 1 to } \\
\text { no. } 30 \text { ) }\end{array}$} & $\mathrm{n}$ & 8 & 8 & 6 & 8 \\
\hline & $\%$ & $27 \%$ & $27 \%$ & $20 \%$ & $27 \%$ \\
\hline \multicolumn{6}{|c|}{ Median of elicitations } \\
\hline \multirow{2}{*}{$\begin{array}{l}\left(\mathbf{P}_{\mathrm{f}}\right) \\
\text { (no. } 1 \text { to } \\
\text { no. } 30)\end{array}$} & $\mathrm{n}$ & 7.67 & 7.67 & 8.83 & 5.83 \\
\hline & $\%$ & $26 \%$ & $26 \%$ & $29 \%$ & $19 \%$ \\
\hline
\end{tabular}

Table 2. Distribution of the true values of the calibration variables (no.1-30) in the interquantile intervals elicited by the experts and the median of the elicitations.
The percentage of the true values contained in the range $[5 \%, 95 \%]$ varies from $40 \%(17 \%+23 \%)$ for expert no. 3 to $66 \%(33 \%+33 \%)$ for expert no. 2 , which is considerably lower than the target percentage of $90 \%$. The distribution of the median of the elicitations of expert opinions contains $55 \%$ of the true values in the uncertainty interval $[5 \%, 95 \%]$, which is also considerably lower than the target percentage of $90 \%$. This means that a large number of true values lie outside the uncertainty intervals elicited by the panel experts, reflecting a trend towards overconfidence or under-confidence in the expert judgement.

The distribution of the median of the elicitations of expert opinions contains $26 \%$ of true values in the interval $[0 \%, 5 \%]$, which is higher than target percentage of $5 \%$. This means that a high percentage of the expert evaluations led to overestimated values for the failure probability, reflecting a trend towards under-confidence, and expressing caution in the experts' evaluations. These overestimated evaluations tend towards safety, but can also lead to decisions to carry out expensive levee reinforcement works in excess of their real necessity. On the other hand, the distribution of the mean of the elicitations of expert opinions contains $19 \%$ of true values in the interval $[95 \%, 100 \%]$, which is also higher than the target percentage of $5 \%$. This means that a high percentage of the expert evaluations also gave underestimated failure probability values, reflecting a trend towards overconfidence. These underestimated evaluations do not tend towards safety and lead to overestimating the levee's resistance. The combined presence of large numbers of biases of over- and under-confidence (with nonetheless a greater bias towards under-confidence tending towards safety) demonstrate in a general way the biases intrinsic to expert evaluations.

\subsection{Application of the expert opinion calibration phase}

The result of the expert opinion aggregation phase is represented by a single probabilistic distribution corresponding to the evaluation by the panel of experts. Figure 5 illustrates the result for the 30 calibration variables associated with the theoretical levee crosssections (continuous lines). The true values are indicated by a circle. 


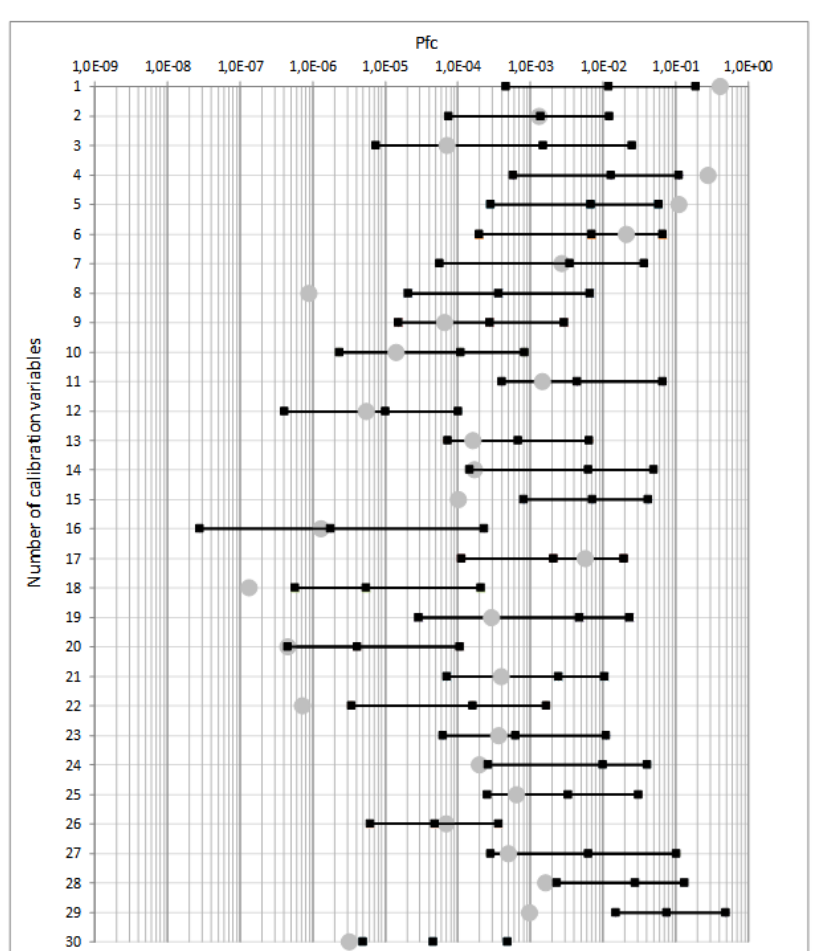

Figure 5. Calibrated and aggregated calibration variables and their uncertainty intervals.

Figure 5 can be interpreted using Table 3 below which presents the distribution of the true values of the calibration variables in the interquantile intervals at the end of the expert opinion aggregation phase:

\begin{tabular}{|c|c|c|c|c|c|}
\hline \multicolumn{5}{|c}{ Calibrated and aggregated elicitations of calibration variables (no.1 to no.30) } \\
\hline $\begin{array}{c}\text { Interquartile } \\
\text { interval }\end{array}$ & {$[\mathbf{0 \%} \% \mathbf{5} \%]$} & {$[\mathbf{5 \%}, \mathbf{5 0} \%]$} & {$[\mathbf{5 0} \%, \mathbf{9 5} \%]$} & {$[\mathbf{9 5} \%, \mathbf{1 0 0} \%]$} \\
\hline $\begin{array}{c}\left(\mathbf{P}_{\mathrm{f}}\right) \\
(\text { no.1 to } \\
\text { no.30) }\end{array}$ & $\mathrm{n}$ & 9 & 15 & 3 & 3 \\
\cline { 2 - 6 } & $30 \%$ & $50 \%$ & $10 \%$ & $10 \%$ \\
\hline
\end{tabular}

Table 3. Distribution of the true calibration values (no.1-no.30) in the interquantile intervals.

At the end of the calibration and aggregation phase we observed that the distributions of the calibrated and aggregated expert opinions contained $60 \%$ of true values in the uncertainty interval $[5 \%, 95 \%]$. Thus, the aggregation and the calibration of expert opinions led to a higher number of true values in the uncertainty interval $[5 \%, 95 \%]$ in comparison to the raw elicitations comprising $55 \%$ of true values in this interval (Table 2). This calibration and aggregation phase therefore improved the quality of the expert evaluations, despite the fact that the correction did not allow reaching the percentage of the ideal target interval (90\%), leaving a still considerable bias of overconfidence or under-confidence following the calibration and aggregation phase.

More specifically, the results obtained following the calibration and aggregation phase showed a substantial reduction of the true values in the overconfidence interval $[95 \% ; 100 \%]$, falling from $19 \%$ (Table 2) to $10 \%$ (Table 3), which was a significant improvement and thus tending to increase the safety of the levees. The evolutions in the interval $[5 \%, 95 \%]$ were slight, changing from $26 \%$ (Table 2) to $30 \%$ (Table 3 ), expressing a small evolution in processing under-confidence during the calibration and aggregation phase.

\subsection{Application and results of the expert panel opinion debiasing phase}

By applying the debiasing approach, the Figure 6 shows the debiased opinions of the expert panel resulting from the application of correction coefficients to the aggregated opinions of the panel of experts:

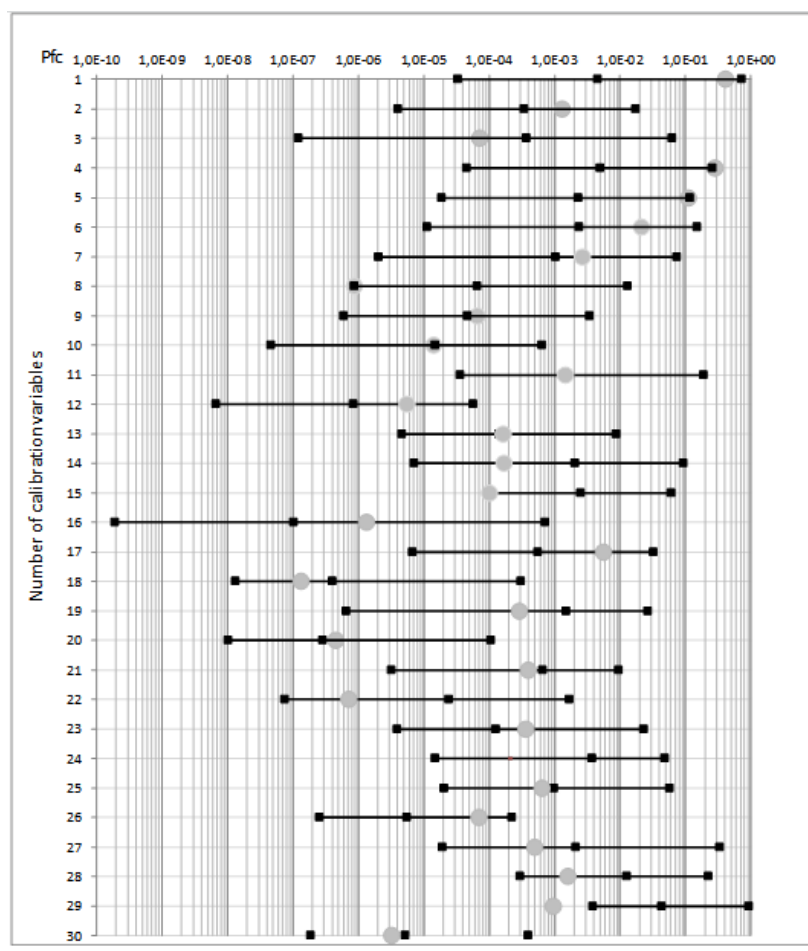

Figure 6. Elicitations of debiased calibration variables of the panel of experts.

Figure 6 is interpreted using table 4 below which presents the distribution of the true values of the calibration variables in the interquantile intervals following the debiasing phase:

\begin{tabular}{|c|c|c|c|c|c|}
\hline \multicolumn{2}{|c|}{$\begin{array}{c}\text { Interquantile } \\
\text { interval }\end{array}$} & {$[0 \%, 5 \%]$} & {$[\mathbf{5} \%, \mathbf{5 0} \%]$} & {$[\mathbf{5 0} \%, \mathbf{9 5} \%]$} & {$[95 \%, 100 \%]$} \\
\hline \multirow{2}{*}{$\begin{array}{c}\left(\mathbf{P}_{\mathrm{fc}}\right) \\
\text { (no.1 to 30) }\end{array}$} & $\mathrm{n}$ & 1 & 14 & 14 & 1 \\
\hline & $\%$ & $3 \%$ & $47 \%$ & $47 \%$ & $3 \%$ \\
\hline
\end{tabular}

Table 4. Distribution of the true values of the calibration variables (no.1 to 30 ) in the debiased interquantile intervals.

At the end of the debiasing phase, we observed that the distributions of the opinions of the expert panel contained $94 \%$ of true values in the uncertainty interval [5\%,95\%]. Thus, the debiasing phase of the opinions of the expert panel permitted reaching the percentage of the ideal target.

The results obtained after the debiasing phase show an optimal reduction of true values in the overconfidence interval $[0 \% ; 5 \%]$, falling from $30 \%$ (Table 3 ) to $3 \%$ (Table 4), indicating a considerable improvement in the quality of the evaluation of the structures. The evolutions in the interval $[95 \%, 100 \%]$ were also considerable, decreasing from $10 \%$ (Table 3 ) to $3 \%$ (Table 4), expressing a marked evolution in the treatment of 
overconfidence bias during the expert opinion debiasing phase, tending towards an improvement in the safety of the structures.

\subsection{Synthesis of the leCAD approach to processing expert judgment}

Figure 7 summarises the evolution of the quality of information provided by expert judgment in the sequence of the different phases of processing the IeCAD approach:

- interval $[5 \%, 95 \%]$ : the distributions of the expert panel opinions contained $55 \%$ of true values following the elicitation phase, then $60 \%$ following the calibration phase, then $94 \%$ following the debiasing phase;

- interval $[0 \%, 5 \%]$ : the distributions of the expert panel opinions contained $26 \%$ of true values following the elicitation phase, then $30 \%$ following the calibration phase, then $3 \%$ following the debiasing phase;

- interval [95\%, 100\%]: the distributions of the expert panel opinions contained $19 \%$ of true values following the elicitation phase, then $10 \%$ following the calibration phase, then $3 \%$ following the debiasing phase.

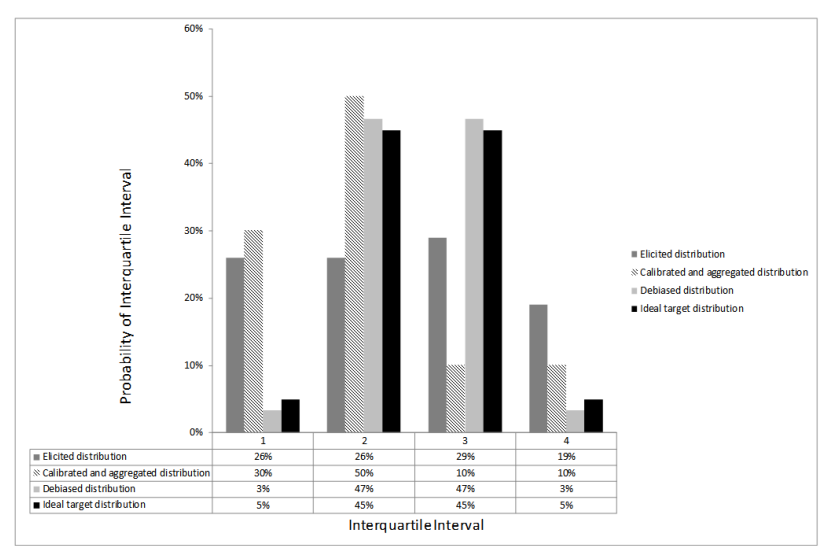

Figure 7. Evolution of the quality of information in the sequence of processing the IeCAD approach.

The expert judgement calibration and debiasing approach was applied to the variables of interest. Figure 8 presents the results on 30 variables of interest corresponding to 10 levee cross-sections studied for 3 failure mechanisms (sliding, internal erosion, scouring).

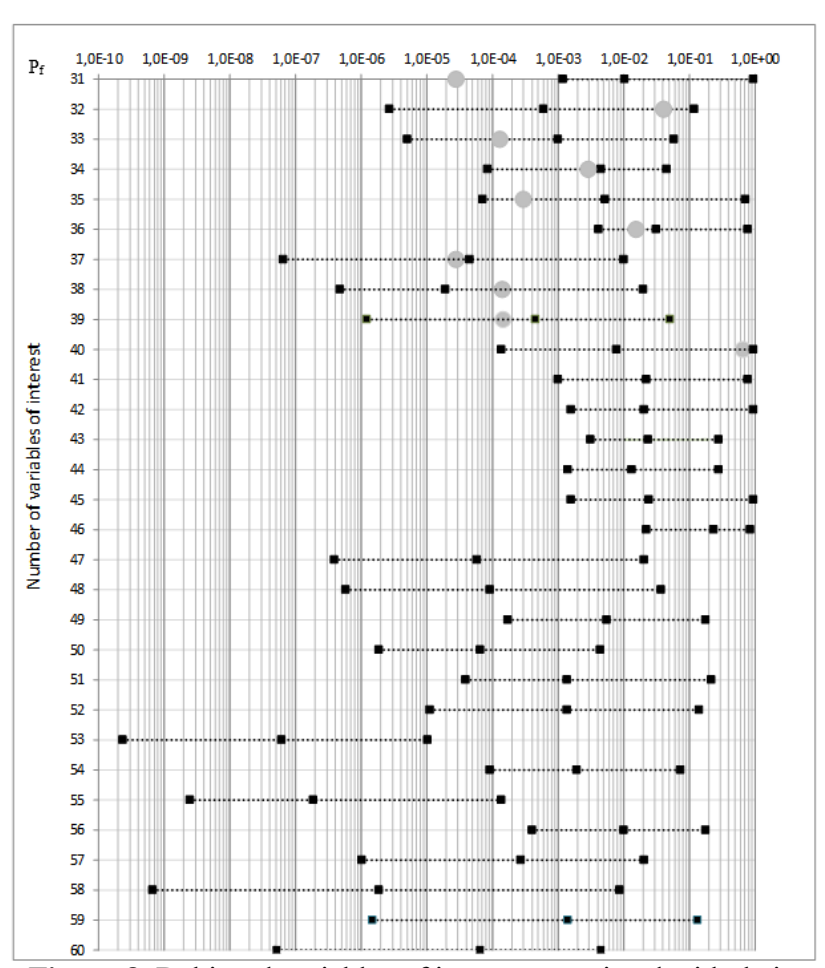

Figure 8. Debiased variables of interest associated with their confidence intervals.

For variables of interest no. 31 to 40 relating to the sliding mechanism, Table 5 shows the distribution of the true values in the inter-quantile intervals, calibrated and aggregated on the one hand, and aggregated and debiased on the other.

\begin{tabular}{|c|c|c|c|c|c|}
\hline $\begin{array}{c}\text { Inter-quantile } \\
\text { interval }\end{array}$ & {$[\mathbf{0} \%, \mathbf{5} \%]$} & {$[\mathbf{5} \%, \mathbf{5 0} \%]$} & {$[\mathbf{5 0} \%, \mathbf{9 5} \%]$} & {$[\mathbf{9 5} \%, \mathbf{1 0 0} \%]$} \\
\hline \multicolumn{7}{|c|}{ Calibrated and aggregated variables } \\
\hline \multirow{2}{*}{$\begin{array}{c}\left(\mathbf{P}_{\mathbf{f}}\right) \\
(\text { no.31to } 40)\end{array}$} & $\mathrm{n}$ & 2 & 5 & 2 & 1 \\
\hline \multicolumn{7}{|c|}{ Calibrated, aggregated and debiased variables } \\
\hline $\begin{array}{c}\left(\mathbf{P}_{\mathbf{f}}\right) \\
\text { (no.31to 40) }\end{array}$ & $\mathrm{n}$ & 1 & 6 & 3 & $10 \%$ \\
\cline { 2 - 6 } & $10 \%$ & $60 \%$ & $30 \%$ & 0 \\
\hline
\end{tabular}

Table 5. Distribution of the true values of the calibration variables (no.1 to 30 ) in the debiased interquantile intervals.

At the end of the debiasing phase, we observed that the distributions of the debiased expert panel opinions contained $90 \%$ of true values in the uncertainty interval $[5 \%, 95 \%]$. Thus the expert panel opinion debiasing phase permitted reaching the ideal target interval.

In detail, the results obtained after the debiasing phase show a reduction of the true values in the under-confidence interval $[0 \% ; 5 \%$ ], falling from $20 \%$ (Table 5 - calibration and aggregation phase) to $10 \%$ (Table 5-debiasing phase), which is a considerable improvement, tending towards increased reliability of the structures. The evolutions in the interval $[95 \%, 100 \%]$ are equally considerable, dropping from $10 \%$ (Table 5 - calibration and aggregation phase) to $0 \%$ (Table 5- debiasing phase), reflecting a substantial change in the treatment of the overconfidence bias during the expert opinion debiasing phase. These evolutions of overconfidence bias are positive since they tend towards greater reliability for the structure.

The correction provided by debiasing therefore appears significant, demonstrating the advantage of applying a full 
treatment including calibration-aggregation and debiasing to the evaluations performed by the panel of experts for failure probabilities. By way of illustration, Figure 8 shows the treatment of the variables of interest for the internal erosion (no.41-50) and scouring (no.51-60) failure mechanisms for which no limit state models were available.

\subsection{Discussion}

The results obtained in the case study show that the calibration-aggregation phase as well as the debiasing phase of the developed approach allow to improve the results obtained from expert elicitations.

The quality of the final result is logically conditioned by the quality of the experts. Logically more experienced experts can result in better evaluation of the levee failure probability, statistically. This would be reflected in particular in the width of the uncertainty intervals ([5\%, 95\%] in the case study): the better the experts, the narrower the interval (leading to a more precise result); on the contrary, with less experienced experts, the interval obtained would be wider (leading to an imprecise result).

In the case study, the panel was composed of 6 experts, which is a rather high number for a risk analysis study of river levee (Peyras et al., 2012). These experts had different experiences in the concerned disciples (geotechnics, civil engineering, hydraulics) and also different levels of qualification (junior, confirmed and senior). Thus, there is necessarily a dispersion in their assessments.

This paper did not study the influence of the number of experts on the results. In the case study, a panel of 6 experts was selected in order to reproduce a panel of experts as implemented in the river dike risk analysis studies (typically 3 to 6 experts - see Peyras et al., 2012). However, an increase in the number of experts would not necessarily lead to an improvement in results: for example, it can be expected that the quality of results would be better if the number of experts were reduced by keeping the most qualified experts. Thus, the professional qualification of experts would have a greater influence on the results in relation to the number of experts.

\section{Conclusion}

Regarding the field of river levees, the lack of data on structures, the uncertainties that impair them and the lack of consensus in the state of the art for the limit states related to internal erosion and scouring failure mechanisms, reduce the opportunity of using statistical and probabilistic methods for determining their failure probability using mechanistic-probabilistic approaches. This makes expert judgement essential for evaluating the failure probabilities of levees with respect to different failure mechanisms.

However, the presence of biases, which will impair the opinions of experts, is a considerable drawback when calling on expert judgement in a risk analysis study. Consequently, our study proposed an approach for evaluating the failure probabilities of levees based on expert judgement and which includes the treatment of the latter.

The approach proposed permits eliciting, calibrating, aggregating and debiasing expert evaluations. The application of this methodological approach provided significant advantages:

- combining expert opinions based on calibration scores,

- estimating a quantitative uncertainty on the final failure probability obtained using an uncertainty interval,

- identifying the best expert elicitations and assigning a calibration weighting according to their pertinence and precision,

- treating the presence of over- and under-confidence bias quantitatively in order to obtain a final debiased failure probability.

At the end, the methods proposed use the expert judgment for estimating the levee failure probability (the estimated or the subjective probability of failure), constituting an expert estimate of the actual levee failure probability.

On the basis of this work, our research will be continued by testing our method to several dike case studies so as to continue validating the robustness of the approach. At the end, we plan to use the IeCAD approach in an operational framework to evaluate the probabilities of failure mechanisms (internal erosion and scouring) in risk analysis studies.

\section{References}

1. Ciria, MEDE \& USACE (2013), The International Levee Handbook. London: CIRIA.

2. Clemen, R.T., Lichtendahl, K.C. (2002), Debiasing expert overconfidence: a Bayesian calibration model. PSAM6, San Juan, Puerto Rico

3. Cooke, R. (1991), Experts in uncertainty: opinion and subjective probability in science. Oxford University Press, $336 \mathrm{p}$.

4. Hathout M., Peyras L., Carvajal C., Diab Y., Vuillet M. (2020). Development of an approach to evaluate the failure probabilities of river levees based on expert judgement: Application to a case study. J Flood Risk Manag, 18p. DOI: 10.1111/jfr3.12603

5. Koehler, J., Harvey, N. (2004), Blackwell Handbook of judgment \& Decision Making, Blackwell Publishing, UK, 675p.

6. Kolen B., Slomp R. \& Jonkman S.N. (2013), The impacts of storm Xynthia February 27-28, 2010 in France: lessons for flood risk management. J Flood Risk Manag, 6, 261-278. doi:10.1111/jfr3.12011.

7. Lichtendahl, K.C Jr., Grushka-Cockayne, Y. et Winkler, RL. (2013), Is it better to average probabilities or quantiles? Management Sci. 59:1594-1611.

8. Mériaux, P. \& Royet, P. (2007), Surveillance, Maintenance and Diagnosis of Flood Protection Dikes - A Practical Handbook for Owners and Operators. Ed. Quae. 
9. Mouyeaux, A., Carvajal, C., Bressolette, P, Peyras, L., Breul P., Bacconnet C (2018). Probabilistic stability analysis of an earth dam by SFEM based on field data. Computers and Geotechnics. 101 (2018) 34-47. Doi.org/10.1016/j.compgeo.2018.04.017

10. Peyras L., Carvajal C., Felix H., Bacconnet C., Royet P., Becue JP., Boissier D. (2012). Probability-based assessment of dam safety using combined risk analysis and reliability methods - application to hazards studies, European Journal of Environmental and Civil Engineering, DOI:10.1080/19648189.2012.672200

11. Peyras, L., Tourment, R., Vuillet, M., Beullac, B., Delaunay, C., Bambara, G. (2015), Development of an expert-led GIS-based approach for assessing the performance of river levees: the Digsure method and tool, J Flood Risk Manag. DOI: 10.1111/jfr3.12178

12. Peyras, L., Royet P. \& Boissier, D. (2006), Dam ageing diagnosis and risk analysis: development of methods to support expert judgment. Can Geotechnical J, 43, pp. 169-186.

13. Simm, J.D., Wallis M., Smith, P., Deniaud, Y., Tourment, R., Veylon, G., Durand, E., McVicker, J., Hersh-Burdick, R. \& Glerum, J. (2012), The significance of failure modes in the design and management of levees - a perspective from the International Levee Handbook team. In eds. FloodRisk Rotterdam Netherlands, 20th-22nd November 2012.

14. Van der Meij, R., Van den Ham, G.A., Morris, M., Lhomme, J., Tourment, R. \& Maurel, P. (2012), Combining different data sources for assessment of urban flood defences. In eds. Floodrisk Rotterdam Netherlands, 20th-22nd November 2012.

15. Vick, Steven, G. (2002), Degrees of Belief: Subjective Probability and Engineering Judgment. American Society of Civil Engineers. USA, 455p.

16. Vuillet, M., Peyras, L., Carvajal, C., Serre, D., Diab, Y. (2013), Levees performance evaluation based on subjective probability, European Journal of Environment and Civil Engineering, Volume 17, issue 5, pp 329-349. 